\title{
Expression Analysis of Interferon Beta in HEK293T Cells Using Real-Time PCR and Protein Tests
}

\author{
Raheleh Norouzi ${ }^{1}$, Zohreh Hojati ${ }^{1^{*}}$ \\ ${ }^{1}$ Division of Genetics, Department of biology, Faculty of Sciences, University of Isfahan, Isfahan, Iran.
}

Received: 12 Aug 2016

Revised : 18 Sep 2016

Accepted: 5 Oct 2016

Corresponding Author: Zohreh Hojati

Division of Genetics, Department of biology, Faculty of Sciences,

University of Isfahan, Isfahan, Iran.

Phone: +98311-7932478

E-mail: z.hojati@sci.ui.ac.ir

\begin{abstract}
Background: Interferons are some kind of natural cytokines which express in response to a variety of antigens including viral RNA, bacterial products, and tumor proteins. Interferon beta is used in the treatment of autoimmune diseases such as multiple sclerosis. Moreover, this drug inhibits cellular proliferation as well as angiogenesis and as a result, helps to cure cancer. In this research, in addition to cloning the interferon-beta gene along with conserved kozak sequence after the strong eEfla promoter in the pBud.CE4.1 vector, the expression of this recombinant gene was compared to basal expression in HEK293T cell line using real-time PCR, SDS PAGE and western blot tests.

Materials and Methods: In the beginning, the interferon-beta gene was amplified from the pSVM dhfr vector containing the gene, using primers including BgIII and $\mathrm{KpnI}$ restriction sites as well as conserved kozak sequence. Then the duplicated gene was digested and inserted in the linear pBud.CE4.1 vector. After ensuring entry of the gene using RFLP, colony PCR and sequencing, the recombinant vector was transfected into E. coli TOP10 competent bacteria. After that, amplified recombinant vector was extracted and transfected into HEK293 cell line. Results: The expression of interferon-beta cloned in pBud.CE4.1 vector showed a 79.9-fold increase, in comparison with the basal expression in HEK293T cell line. Moreover, non-recombinant vector transfection has increased the expression of interferon-beta up to 2.87 times in the cell line that is probably due to the existence of the viral promoter in the vector.

Conclusion: Real-time PCR and protein test results showed that recombinant beta interferon gene had been successfully expressed in HEK293T cell line. In order to produce more of this protein, optimization of various conditions are required for the HEK293T cell line.
\end{abstract}

Keywords: HEK293T cells; Interferon-Beta 1a; Real-time PCR; SDS PAGE; Western blot

Please cite this article as: Norouzi R, Hojati Z. Expression Analysis of Interferon Beta in HEK293T Cells Using Real-Time PCR and Protein Tests. Res Mol Med. 2016; 4 (4): 15-21

\section{Introduction}

Interferons (IFN) are key mediators of the immune system and a group of secretory cytokines with low molecular weight, which possess a wide range of biological effects including anti-tumor, immunomodulatory, and cell differentiation (1). These proteins have an important role in protecting the host against foreign organisms, unknown antigens as well as types of abnormal cells and due to antiviral, antitumor and anti-inflammatory properties, they have been regarded as a medicine, traditionally (2). Expression and synthesis of IFNs increase in all cell types after viral infection. Virus entry into the cell induces the expression of IRF-1 (interferon regulatory factor 1) which is a DNA- binding protein. IRF-1 increases the expression of IFN via binding to the interferon's upstream regulatory sequence. In addition, IFNs induce the expression of several different genes which interfere with the process of 
viral replication. Moreover, IFNs are effective factors in many cancer tumors. Anti- tumor effects of IFNs include: direct impact on cell cycle and replication as well as induction of apoptosis. IFNs through activating immune system, increase the expression of major histocompatibility complex (MHC), cytotoxic T-cell and natural killer cells (NKC) as well as induction of the monocyte function, indirectly $(4,5)$. IFNs are generally divided into three groups based on amino acid sequence. The group I includes IFNs $\alpha, \beta$, $\varepsilon, \delta, \omega, \kappa$ and $\tau$ while the group II has only one member called $\gamma$ interferon. The group III IFNs have been identified recently and include $\lambda 1, \lambda 2$ and $\lambda 3$ which play an important role in direct response to viral infections. Another classification for IFNs is based on their cell origin. According to this classification IFNs could be divided into three groups: IFN- $\alpha$ with leukocyte cell origin, which is called leukocyte interferon, interferon- $\beta$ derived from fibroblast cells that is called fibroblast interferon and interferon- $\gamma$ with its origin in lymphocytic cells which is called immune interferon $(6,7)$.

Nowadays, two completely different strategies are used to produce different drug forms of recombinant interferon- $\beta$. IFN $\beta$-Ia form is the generic name for drugs that are produced in $\mathrm{CHO}$ cell line and IFN $\beta-\mathrm{Ib}$ is the general name for medications that come into production using $E$. coli expression system. In the beginning, recombinant interferon- $\beta$ production in $E$. coli cells had some problems such as the formation of dimer and oligomer between IFNs which made their purification and separation very hard and timeconsuming (8-10). In addition, it was found that interferon- $\beta$ by microbial methods always had lower specific activities than the natural form. With regard to these problems in biopharmaceutical industry, mammalian cell culture systems are preferred (11).

In this research, in addition to cloning the Interferonbeta gene along with conserved kozak sequence after the strong eEfla promoter in the pBud.CE4.1 vector, the expression of this recombinant gene in comparison to basal expression in HEK293T cell line was done using real-time PCR, SDS PAGE and western blot tests.

\section{Materials and methods}

Primer design and construction of recombinant pBud.IFN $\beta$ - $1 a$

In order to study the expression interferon- $\beta$ 's mRNA, pSVMdhfr vector containing human IFN- $\beta$ was used as a template for PCR. Specific primers were designed using Oligo v7 (Molecular Biology Insight, USA) in a way which allowed replication of human IFN- $\beta$ gene region from pSVMdhfr vector. Recognition sites for KpnI and BglII enzymes were designed in forward and reverse primers in order to clone the gene fragment in target vector. Using GG nucleotides before KpnI restriction site in forward primer and TC nucleotides after the BglII restriction site in reverse primer, allowed for better identification of restriction sites by the enzymes. Reverse primer was designed in such a way that the TGA termination codon deleted due to providing the possibility of tracing synthesized protein using HisTag antibody. For this reason and to preserve the open reading frame of the gene as well as the expression of histidine sequence, dinucleotide GA was inserted before the kpnI restriction site. One of the important factors that affect translation is the existence of proper start codon surrounding sequence called Kozak sequence. The amount of translated protein depends on the power of Kozak sequence. The Kozak sequence of the IFN- $\beta$ gene has several differences in some positions compared with the conserved sequence form. The Kozak consensus sequence is GCCRCCAUGG in which R represents a purine base. The Kozak sequence of the interferon- $\beta$ is GUCAACAUGA and to approximate this sequence to Kozak consensus sequence, two nucleotides in the -2 and -5 positions -with regard to transcription start site- should be changed to $\mathrm{C}$ nucleotide. Therefore GCCACCATGACC nucleotide sequence related to conserved Kozak sequence, was inserted after the $\mathrm{kpnI}$ restriction site in forward primer. The following primers were used for PCR amplification:

FP IFN $\beta$ : 5'-GGGGTACCGCCACCATGACCAAC AAGTGT-3'

RP IFN $\beta$ : 5'-GAAGATCTTCGTTTCGGAGGTAA CCTG - $3^{\prime}$

The conditions of PCR were as follows: $95^{\circ} \mathrm{C}$ for 6 min and $94{ }^{\circ} \mathrm{C}$ for $30 \mathrm{sec}, 60^{\circ} \mathrm{C}$ for $30 \mathrm{sec}$, and $72^{\circ} \mathrm{C}$ for $50 \mathrm{sec}$ for a total of 35 cycles, and finally $10 \mathrm{~min}$ at $72{ }^{\circ} \mathrm{C}$. Then the amplified segment was purified using gel extraction kit (Thermo Scientific, USA). At the same time, the pBud.CE4.1 vector became linear by kpnI and BglII enzymes. Finally, the amplificated segment was cloned into the linear vector using T4 ligase. Recombinant vector was transformed into $E$. coli. TOP10 bacteria via standard heat shock method. The selection of colonies containing recombinant vector was done on the LBA plate which included 50 $\mu 1 / \mathrm{ml}$ zeocin antibiotic. Obtained colonies were screened via colony PCR and then plasmid extraction was accomplished. RFLP analysis as well as DNA sequencing were done using Bgh Rev universal primers of Iran's Fazabiotech Company.

\section{Cell culture, transfection and RNA extraction}

After confirming the structure of recombinant vector, the HEK293T cell line transfection was done using Lipofectamine ${ }^{\circledR}$ LTX plus ${ }^{\mathrm{TM}}$ reagent (Invitrogen, USA). After 48 hours, the cells were washed with 
PBS buffer, and then were separated from the flask surface using $0.05 \%$ trypsin. After cell separation, the cells were transferred to a falcon and centrifuged for $5 \mathrm{~min}$ at $1700 \mathrm{rpm}$. After obtaining the cell sediment, RNA extraction procedures were carried out according to Qiagen kit instruction. In order to evaluate the quantity of extracted RNA, the spectrophotometer instrument was used and to verify the quality of extraction, RNA samples were loaded on $2 \%$ agarose gel wells. DNase treatment using DNase I kit (Thermo Scientific- USA) was used for removing DNA contamination.

\section{Real-time PCR}

The cDNA synthesis reaction was performed by adding the following compounds: $4 \mu$ buffer X5 of RT enzyme, $1 \mu 1$ of three inhibitors of RNase, RT enzyme, random hexamer primer, $2 \mu 1$ of dNTP mix $10 \mathrm{mM}, 2$ microgram of RNA and the total volume reaction with nuclease-free water was brought to 20 $\mu 1$. In the next step, specific primers for interferon- $\beta$ and eEflal housekeeping gene for RT-PCR were designed using oligo v7 and AlleleID v8.7. At first, in an attempt to determine the best annealing temperature for the primers, the temperature gradient was performed in the range of 50 to $57^{\circ} \mathrm{C}$. After determining the best conditions for amplification, real-time PCR was performed. For accurate investigation of real-time PCR results, insurance of proper bands formation and the absence of dimer primer, samples were analyzed on $2 \%$ agarose gel.

\section{Protein studies}

Once again for protein studies, cell culture and transfection of HEK293T cell line were repeated using Lipofectamine transfection kit. Protein was extracted from two phases of cell culture as well as cell lysis of transfected cells with the recombinant vector. To investigate the possibility of the presence of secretory interferon- $\beta$ in the environment, untransfected control cells and transfected cells with non-recombinant vector were carried out using TRIzol of Life Technology Company according to manufacturer's instructions with catalog number 155996-026. The concentration of obtained proteins were calculated using Bradford standard curve and at each stage of protein test analysis, an equal concentration of samples were used.

\section{SDS-PAGE and western blot}

In order to perform semi-quantitative analysis and comparative studies, 60 micrograms of obtained protein samples (so that the comparison could be made with equal amount of all samples) from cell lysis and cell culture medium were taken for SDSPAGE test. After preparation, all samples were loaded on $12 \%$ polyacrylamide gel. The gel was then stained using Coomassie Brilliant Blue. In order to carry out western blot analysis, after electrophoresis in polyacrylamide gel, protein samples were transferred to a PVDF membrane during the wet blotting reaction. Finally, protein bands were detected using a conjugated antibody with HPR enzyme and the TMB solution as the enzyme substrate.

\section{Statistical analysis}

Statistical analysis was performed via IBM SPSS version 21 through nonparametric test.

\section{Results}

\section{Production of $p$ Bud. IFN $\beta$-1 a construct}

PCR reaction on the pSVMdhfr- vector containing the IFN- $\beta$ gene was performed using the designed primers and a product with 564 bp length was amplified. After the cloning process in pBud.CE4.1 vector, digestion of the recombinant vector with $\mathrm{KpnI}$ and BglII enzymes was performed and exit of $564 \mathrm{bp}$ IFN- $\beta$ segment, confirmed the correct entry of IFN- $\beta$ to the vector (Figure 1).

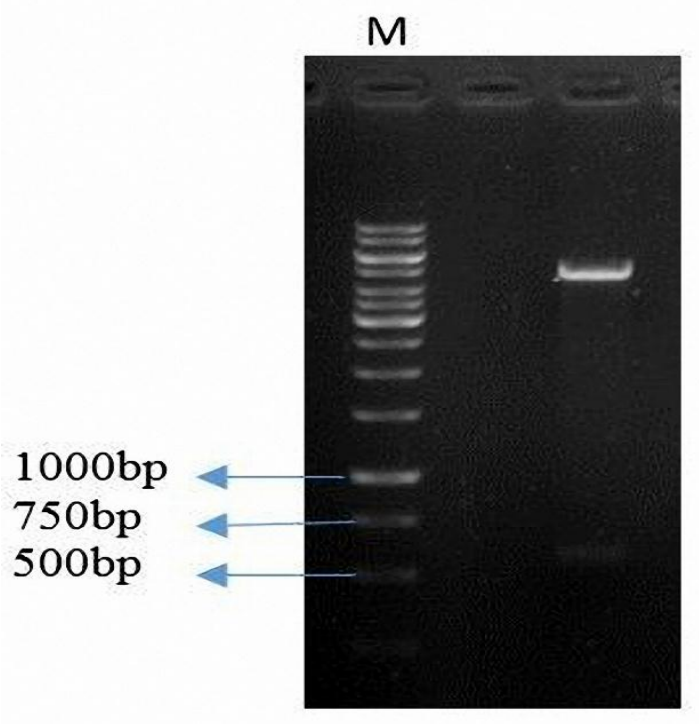

Figure 1. Double enzyme digestion on the recombinant vector.

Double enzyme digestion leads to the production of linear vector with 4595 bp and IFN- $\beta$ gene with 564 bp length. The letter $M$ represents the molecular weight marker (Fermentase Company). The size of segments is based on bp.

Results of pBud.IFN $\beta$-1a transfection into the HEK293T cell line

The recombinant plasmid was transfected into HEK293T cell line using Lipofectamine kit (Invitrogen, USA). In addition, in a separate reaction, non-recombinant vector was transfected into the 
cells. In another flask, un-transfected HEK293T cells

A
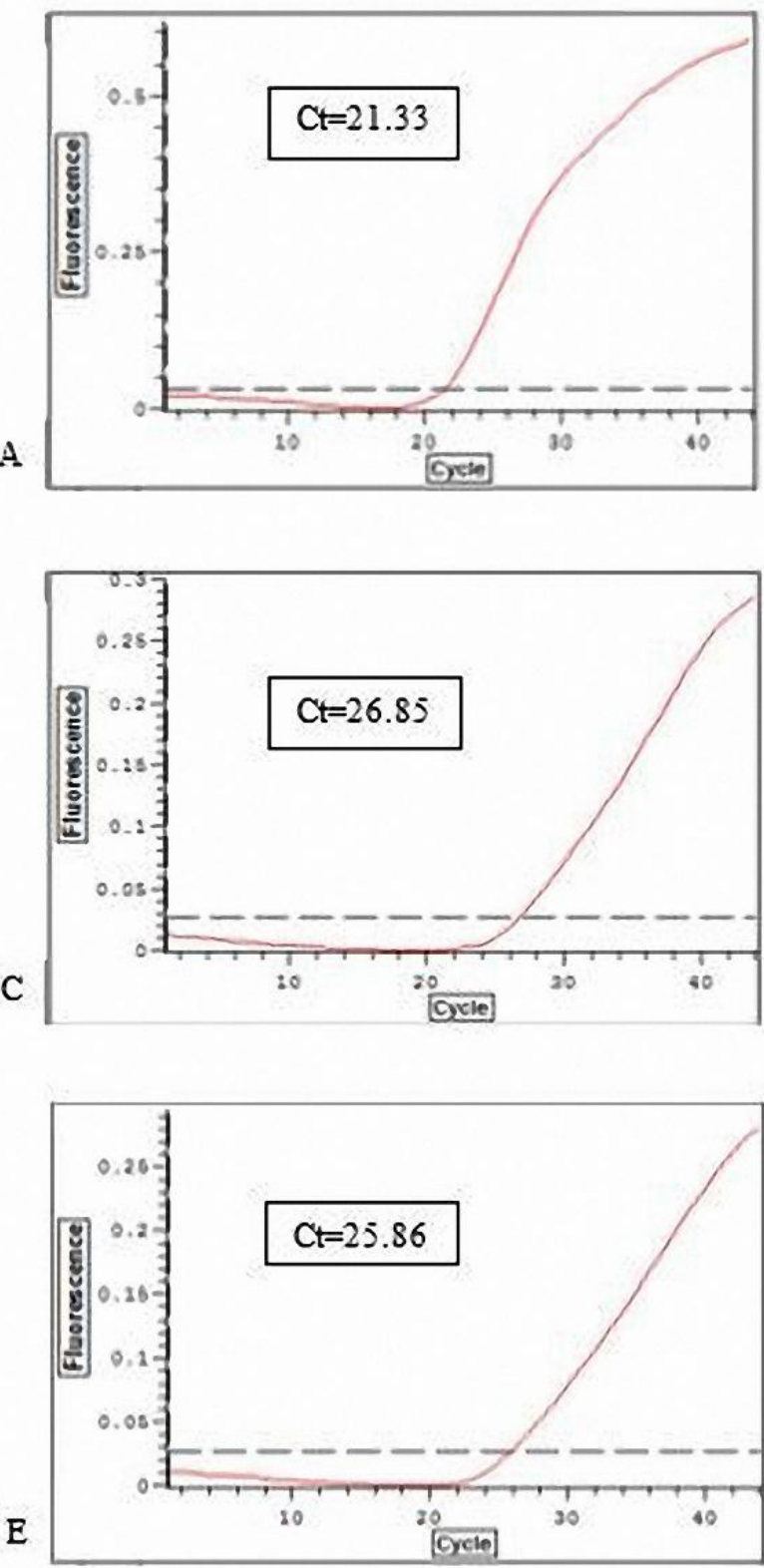

were cultured as control cells.

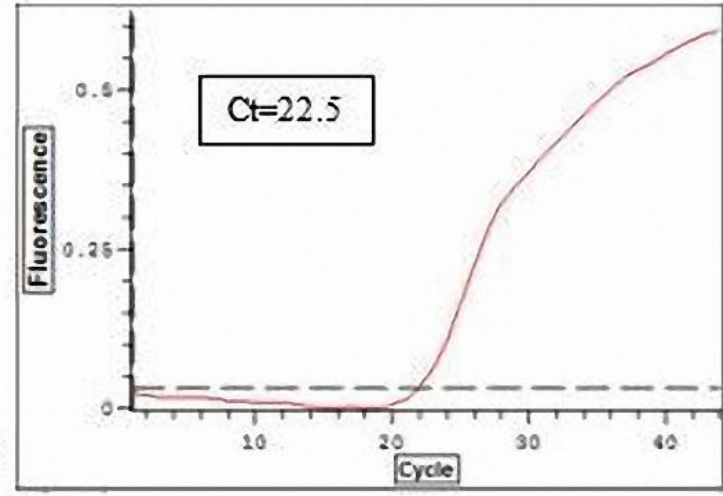

D

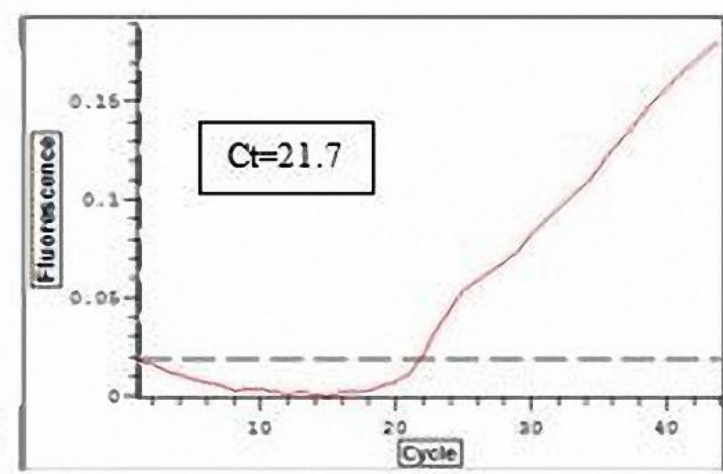

F

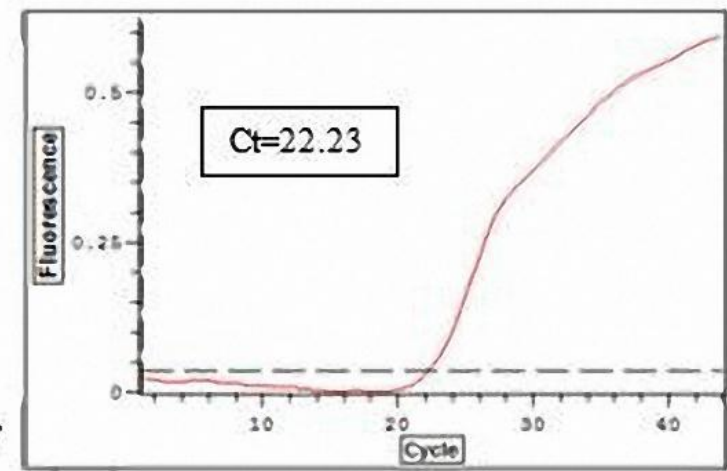

Figure 2. The amplification curve of IFN- $\beta$ gene in real-time PCR.

A) Amplification curve of IFN- $\beta$ gene's cDNA. B) Amplification curve of eEf1a1 control gene on IFN- $\beta$ 's cDNA sample. C) Amplification curve of IFN- $\beta$ gene on cDNA of un-transfected cells. D) Amplification curve of eEflal control gene on cDNA of un-transfected cells. E) Amplification curve of IFN- $\beta$ on cDNA of transfected cells with non-recombinant vector. F) Amplification curve of eEfla1 gene on cDNA of transfected cells with non-recombinant vector.

\section{Real-time PCR results}

Gradient PCR showed that $55^{\circ} \mathrm{C}$ was the optimum temperature for simultaneous amplification of IFN- $\beta$ and eEfla1. Figure 2 shows the amplification curves of the target gene's cDNA as well as the housekeeping gene during real-time PCR.

\section{Protein test results}

To evaluate the production of recombinant IFN- $\beta$, the samples were surveyed on $12 \%$ acrylamide gels (Figure 3). Final confirmation of protein production was done using the western blot method. Results are shown in Figure 4.

The size of protein molecular weight marker is based on $\mathrm{kDa}$. The L letter represents extracted protein from the cell lysis phase and the $\mathrm{S}$ letter represents protein extracted from the cell culture medium phase. $\mathrm{P}$ refers to the extracted protein from the transfecte 
cells with non-recombinant vectors and $\mathrm{UN}$ is the symbol of extracted protein from un-transfected control cells.

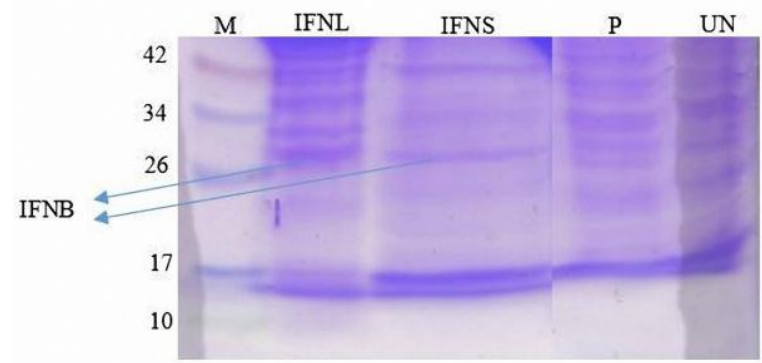

Figure 3. Separation of protein samples from cell lysis and cell extract phases using $12 \%$ polyacrylamide.

The size of protein molecular weight marker is based on $\mathrm{kDa}$. The band intensity of extracted protein from cell lysis phase $(\mathrm{L})$ is stronger than the band intensity of extracted protein from cell culture medium $(\mathrm{S})$.

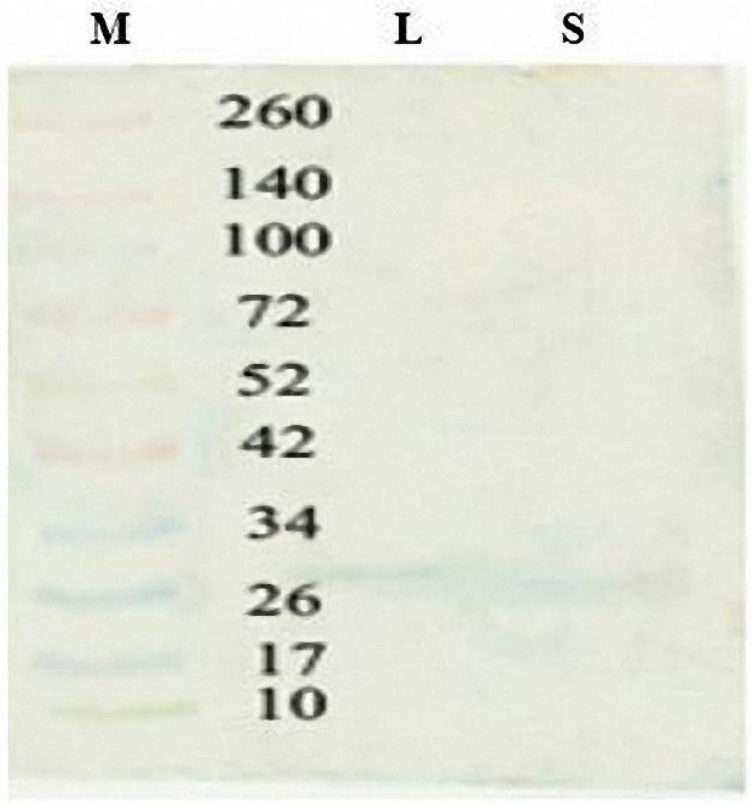

Figure 4. Analysis of produced IFN- $\beta$ protein in HEK293T cell line using the western blot method.

\section{Discussion}

Cytokines are the active cellular factors that are produced by different cell types in response to a wide range of stimulus factors. Effects on cellular mechanisms and changes in cellular behavior are the notable features of cytokines. IFNs have been introduced as the first group of cytokines. IFN- $\beta$ has a wide range of functions such as induction of antiinflammatory activity as well as modulating the immune system which leads to mitigation of symptoms and relative treatment of autoimmune diseases such as multiple sclerosis (MS), and therefore is one of the most common drugs used for this disease (12). Studies in this field have shown that the production of IFN- $\beta$ by immune cells decreased in patients with MS (13). Similarly, the amount of related products to IFN- $\beta$ reduced due to decreased expression of target genes of IFN- $\beta$. Moreover, IFN- $\beta$ has a regulatory effect on its own expression leading to stimulation and up-regulation of IFN- $\beta$ gene; but in MS patients this phenomenon rarely has been observed. This issue shows the importance of producing IFN- $\beta$ as a recombinant form with high level of expression and stability. Studies have shown that IFN- $\beta$ has an anti-tumor activity in many human cancers. Some part of the IFN- $\beta$ 's anti-tumor activity is related to its role in angiogenesis inhibition. IFN- $\beta$ causes suppression of angiogenesis and tumor growth through the inhibition of cytokines that induce angiogenesis as well as inhibition of endothelial cell differentiation (14).

Nowadays, two analogs of IFN- $\beta$ are used as drugs in the treatment of MS (15). The first type is the nonglycosylated protein called IFN $\beta-1 b$ produced by $E$. coli and is currently on the market under the brand name BetaSeron. The glycosylated form of the protein is called IFN $\beta-1 \mathrm{a}$ and the trade names of this protein are Avonex and Rebif, which are both expressed in Chinese hamster ovary $(\mathrm{CHO})$ cell line. IFN $\beta-1 b$ has lower efficiency compared to glycosylated form and therefore higher dose of this drug should be prescribed (16). Continuous injection of the drug stimulates the production of neutralizing and non-neutralizing antibodies against IFN- $\beta$ which inhibit the biological effects of IFN and reduce the influence of the medication. These factors reduce the therapeutic efficiency and cause the incidence of side effects in patients, including flu-like symptoms, liver damage, white blood cells and platelet reduction, depression, and headache (17). To overcome these problems, other expression hosts such as yeasts, filamentous fungi, insect cells and plant cells can be used; but due to different glycosylation patterns, their uses are also associated with some limitations (18).

\section{Conclusion}

Nowadays, recombinant DNA technology and production of recombinant proteins are taken into consideration in many areas of biotechnology. Cloning of factors and proteins related to different biological processes has particular importance in the areas of research and treatment. IFN- $\beta$ protein plays an important role in the treatment of many autoimmune diseases such as multiple sclerosis, viral infection, inhibition of cell proliferation, angiogenesis and cancer (2). Due to the transient expression of this protein in fibroblast cells, more production of IFN protein is desired. 
In this study, the expression of recombinant plasmid containing IFN- $\beta$ gene in HEK293T cell line was evaluated using real-time PCR $(\mathrm{RQ}=2-\Delta \Delta \mathrm{Ct}$ method, $2-\Delta \Delta \mathrm{Ct}=2-[\mathrm{Ct} \quad(\mathrm{IFN} \beta)-\mathrm{Ct}(\mathrm{eEfl} \alpha 1)$ Transfected cells] - [Ct (IFN $\beta)-C t$ (eEf1 $\alpha 1)$ Un-transfected cells) (19)) and the result was confirmed with the outcomes of western blot and SDS-PAGE protein analysis.

After real-time PCR reaction and analyzing the amplification curves of IFN- $\beta$ and eEflal (housekeeping gene), the average of $\mathrm{Ct}$ (after three times repetition of each reaction) was calculated. The difference between eEflal's and IFN- $\beta$ 's $\mathrm{Ct}$ was evaluated for each sample $(\Delta \mathrm{Ct})$. Then the calculated $\Delta \mathrm{Ct}$ for the sample was subtracted from the $\Delta \mathrm{Ct}$ of untransfected control cell in order to calculate $\Delta \Delta \mathrm{Ct}$. The results indicated $\mathrm{p}<0.05$. Based on the results, the expression of transfected IFN- $\beta$ showed a 79.9fold increase compared to basal expression in HEK293T cell line (Table1). Moreover, nonrecombinant vector transfection increased the basal expression of IFN- $\beta 2.87$ folds. As a result, it could be justified that the CMV viral promoter in the selected vector was recognized as a foreign agent in the target cell and therefore IFN- $\beta$ had been produced against the alien agent. Protein tests have shown successful expression of the recombinant IFN- $\beta$ protein in the selected cell line. This could be due to strong eEfla promoter in the vector and creation of conserved Kozak using designed primers for IFN- $\beta$ gene cloning. The speed of translation initiation by ribosomes mostly depends on the existence of a Kozak sequence near the start codon and the amount of produced protein from an mRNA is related to the power of this sequence (20). In this study, by changing the -2 and -5 nucleotides in Kozak sequence and turning it to conserved form, protein expression was significantly increased compared with basal expression. IFN- $\beta$ is a $22 \mathrm{kDa}$ protein that along with linker sequence and histidine sequence would be a 27 $\mathrm{kDa}$ sequence. It can be finally concluded that the expression level of IFN- $\beta$ has increased significantly Significantly due to cloning in pBud.CE4.1 vector containing the strong eEfla promoter. Therefore, the selected cell line and vector possess appropriate features for producing IFN- $\beta$. Moreover, changing Kozak sequence of IFN- $\beta$ and turning it to conserved form, has a significant impact on increasing the level of gene expression.

\section{Acknowledgements}

This study was performed at the University of Isfahan and financially supported by the Graduate Office of the University of Isfahan and Iran National Science Foundation (INSF), (Grant No: 92042856).

\section{Authors' contributions}

NR conception and design, collection and assembly of data, data analysis and interpretation, and manuscript writing. HZ conception and design, provision of study material, data analysis and interpretation, manuscript writing, and final approval of manuscript.

\section{Conflict of interest}

No competing financial and non-financial interests exist.

\section{References}

1. Chelbi-Alix MK, Wietzerbin J. Interferon, a growing cytokine family: 50 years of interferon research. Biochimie. 2007; 89(67):713-8. PMID: 17544197

2. Pang KR, Wu JJ, Huang DB, Tyring SK, Baron S. Biological and clinical basis for molecular studies of interferons. Methods Mol Med. 2005; 116: 1-23. PMID: 16007741

3. Kerr IM, Brown RE. pppA2'p5'A2'p5'A: an inhibitor of protein synthesis synthesized with an enzyme fraction from interferontreated cells. Proc Natl Acad Sci U S A. 1978; 75(1):256-60. PMID: 272640

4. Javed A, Reder AT. Therapeutic role of beta-interferons in multiple sclerosis. Pharmacol Ther. 2006;110(1):35-56. PMID: 16229894

5. Sadler AJ, Williams BR. Interferon-inducible antiviral effectors. Nat Rev Immunol. 2008; 8(7):559-68. PMID: 18575461

6. Fujie H, Numasaki M. Type III Interferons IL-28 and IL-29: Novel Interferon Family Members with Therapeutic Potential in Cancer Therapy: INTECH Open Access Publisher; 2012.

7. Pestka S, Baron S. Definition and classification of the interferons. Methods Enzymol. 1980;78(Pt A):3-14. PMID: 6173606

8. Mark DF, Lin LS. Human recombinant cysteine depleted interferon- $\beta$ muteins. US Patent 4,588,585; 1986.

9. Runkel L, Meier W, Pepinsky RB, Karpusas M, Whitty A, Kimball K, et al. Structural and functional differences between glycosylated and non-glycosylated forms of human interferon- $\beta$ (IFN- $\beta$ ).Pharm Res. 1998;15(4):641-9. PMID: 9587963

10. Zago P, Baralle M, Ayala YM, Skoko N, Zacchigna S, Buratti $E$, et al. Improving human interferon- $\beta$ production in mammalian cell lines by insertion of an intronic sequence within its naturally uninterrupted gene. Biotechnol Appl Biochem. 2009; 52(3):191-8. PMID: 18564058

11. Hossler P, Khattak SF, Li ZJ. Optimal and consistent protein glycosylation in mammalian cell culture. Glycobiology. 2009; 19(9):936-49. PMID: 19494347

12. Dhib-Jalbut $S$, Marks $S$. Interferon- $\beta$ mechanisms of action in multiple sclerosis. Neurology. 2010; 74(1 Supplement 1):S17-S24.

13. Bekisz J, Schmeisser H, Hernandez J, Goldman ND, Zoon KC. Mini ReviewHuman Interferons Alpha, Beta and Omega. Growth Factors. 2004; 22(4):243-51. PMID: 15621727

14. Feng X, Petraglia AL, Chen M, Byskosh PV, Boos MD, Reder AT. Low expression of interferon-stimulated genes in active 
multiple sclerosis is linked to subnormal phosphorylation of STAT1. J Neuroimmunol. 2002; 129(1):205-15. PMID: 12161037

15. Meyer O. Interferons and autoimmune disorders. Joint Bone Spine. 2009; 76(5):464-73. PMID: 19773191

16. Bertolotto A, Deisenhammer F, Gallo P, Sorensen PS. Immunogenicity of interferon beta: differences among products. J Neurol. 2004; 251(2):ii15-ii24. PMID: 15264108

17. Giovannoni G, Munschauer F, Deisenhammer F. Neutralising antibodies to interferon beta during the treatment of multiple sclerosis. J Neurol Neurosurg Psychiatry. 2002; 73(5):465-9. PMID: 12397132
18. Smith GE, Summers M, Fraser M. Production of human beta interferon in insect cells infected with a baculovirus expression vector. Mol Cell Biol. 1983; 3(12):2156-65. PMID: 6318086

19. Pfaffl MW. Quantification strategies in real-time PCR. AZ of quantitative PCR. 2004; 1:89-113. PMID:

20. Kozak M, Evans M, Gardner PD, Flores I, Mariano TM, Pestka $\mathrm{S}$, et al. Structural features in eukaryotic mRNAs that mod. J Biol Chem. 1991; 266:19867-70. PMID: 1939050 